mediating thyroid hormone liberation in thyroid epithelial cells. Endocrinology 137: 1963-1974.

23.Perham, R.N. 2000. Swinging arms and swinging domains in multifunctional enzymes: catalytic machines for multistep reactions. Annu. Rev. Biochem. 69:961-1004.

24.Miklos, G.L. and R. Maleszka. 2001. Protein functions and biological contexts. Proteomics 1:169-178.

25.Boonacker, E.P., E.A. Wierenga, H.H. Smits, and C.J.F. Van Noorden. 2002. CD26/DPPIV signal transduction function, but not proteolytic activity, is directly related to its expression level on human Th1 and Th2 cell lines as detected with living cell cytochemistry. J. Histochem. Cytochem. 50:1169-1177.

26.Hahn, K. and A. Toutchkine. 2002. Live-cell fluorescent biosensors for activated signaling proteins. Curr. Opin. Cell Biol. 14:167-172.

27.Patton, W.F. and J.M. Beechem. 2002. Rainbow's end: the quest for multiplexed fluorescence quantitative analysis in proteomics. Curr. Opin. Chem. Biol. 6:63-69.

28.Lambeir, A.M., P. Proost, C. Durinx, G. Bal, K. Senten, K. Augustyns, S. Scharpe, J. Van Damme, and I. De Meester. 2001. Kinetic investigation of chemokine truncation by CD26/dipeptidyl peptidase IV reveals a striking selectivity within the chemokine family. J. Biol. Chem. 276:29839-29845.

29.Hinke, S.A., J.A. Pospisilik, H.U. Demuth, S. Mannhart, K. Kuhn-Wache, T. Hoffmann, E. Nishimura, R.A. Pederson, and C.H.S. McIntosh. 2000. Dipeptidyl peptidase IV (DPIV/CD26) degradation of glucagon. Characterization of glucagon degradation products and DPIV-resistant analogs. J. Biol. Chem. 275:3827-3834.

30.Lojda, Z., R. Gossrau, and T.H. Schiebler. 1976. Enzyme Histochemistry. A Laboratory Manual. Springer, New York.

31.Hendriks, T. and T.J. Benraad. 1981. On the stability of immunoreactive glucagon in plasma samples. Diabetologia 20:553-557.

32.Marki, F. 1983. Stability of endogenous immunoreactive glucagon (IRG) in animal blood. Horm. Metab. Res. 15:307-308.

33.Kasahara, Y., G. Leroux-Roels, R. Nakamura, and F. Chisari. 1984. Glycylprolyl-diaminopeptidase in human leukocytes: selective occurrence in $\mathrm{T}$ lymphocytes and influence on the total serum enzyme activity. Clin. Chim. Acta 139:295-302.

34.Kahne, T., H. Kroning, U. Thiel, A.J. Ulmer, H.D. Flad, and S. Ansorge. 1996. Alterations in structure and cellular localization of molecular forms of DP IV/CD26 during T cell activation. Cell. Immunol. 170:63-70.

Received 14 May 2003; accepted 16 July 2003.

Address correspondence to Cornelis J.F. Van Noorden, Department of Cell Biology and Histology, Academic Medical Center, Meibergdreef 15, 1105 AZ Amsterdam, The Netherlands. e-mail: c.j.vannoorden@ amc.uva.nl

\title{
Direct retransformation of yeast with plasmid DNA isolated from single yeast colonies using rolling circle amplification
}

\author{
Xiaodong Ding, Anita K. Snyder, Regina Shaw, William G. Farmerie, \\ and Wen-Yuan Song \\ University of Florida, Gainesville, FL, USA
}

BioTechniques 35:774-779 (October 2003)

We have efficiently amplified plasmid DNA from single yeast colonies using rolling circle amplification (RCA). The amplified DNA can be directly used for restriction digestion, DNA sequencing, or yeast transformation. The RCA-based high-fidelity amplification would be useful for plasmid manipulation in a variety of yeast-based systems, particularly for highthroughput analyses.

\section{INTRODUCTION}

Yeast-based systems, such as onehybrid and two-hybrid screening, are powerful tools for studying proteinDNA and protein-protein interactions (1). However, yeast is not ideal for direct analysis of plasmid DNA. Typically, only a small quantity of plasmid DNA can be isolated from yeast cells using conventional procedures. The recovered plasmids are not sufficient for many routine molecular assays, such as restriction digestion, DNA sequencing, and subsequent yeast transformation. Therefore, further propagation of the recovered DNA in bacterial hosts becomes essential. This approach is feasible for low-throughput analyses. When performed in a high-throughput manner, the approach would, however, involve thousands of yeast and bacterial cultures, bacterial transformations, and plasmid DNA preparations from both bacteria and yeast. Such quantities of work are not practical for many research laboratories.

Rolling circle amplification (RCA) is an in vitro DNA amplification technique utilizing the rolling circle mechanism used by bacteria to replicate circular plasmids or viruses (2). By using random hexamer primers and ф29 DNA polymerase, circular DNA templates can be amplified 10,000fold at a constant temperature in a short time period (3). The $\$ 29$ DNA polymerase has the capacity to perform displacement DNA synthesis for more than 70,000 base pairs without disso- ciation from the template and has high proofreading activity to ensure a high fidelity of DNA amplification $(4,5)$. It has been reported that the error rate for this enzyme is $10^{-6}-10^{-7}(6)$.

Here we describe a method to amplify plasmid DNA from single yeast colonies using RCA. The amplified DNA is sufficient for restriction analysis, DNA sequencing, and retransformation of yeast cells for confirmation of the initial yeast two-hybrid interactions.

\section{MATERIALS AND METHODS}

The yeast strain CG-1945 (MATa, ura3-52, his3-200, lys2-801, ade2-101, trp 1-901, leu2-3, 112, gal4-542, gal80538, LYS 2::GAL1 UAS- GAL1 ${ }_{\text {TATA }}-H I S 3$, URA3::(GAL4 17-mers) ${ }_{3}$-Cycl ${ }_{\text {TATA }}$-lacZ, $c y h^{r}$ ) was used in this study. Transformation of the yeast cells was carried out using the Frozen-EZ Yeast Transformation II ${ }^{\mathrm{TM}}$ kit (Zymo Research, Orange, CA, USA) according to manufacturer's instructions (http://www.zymoresearch.com/t2001frame.html). Yeast two-hybrid assays were performed according to the methods described (7). The primers used for DNA sequencing are listed in Table 1.

The RCA reactions were performed using the TempliPhi ${ }^{\mathrm{TM}} 100$ Amplification kit (Amersham Biosciences, Piscataway, NJ, USA). Briefly, 1/3-1/2 of each single yeast colony was picked using a toothpick and suspended into 5 $\mu \mathrm{L}$ of sample buffer (Amersham Biosciences). For enzymatic treatment, $2 \mathrm{U}$ of zymolase (Zymo Research) were added 
Table 1. Primers Used for DNA Sequencing

\begin{tabular}{|ll|}
\hline Primer Name & Sequence \\
\hline GAL4-DB & 5'-TCATCGGAAGAGAGTAG-3' \\
SSO20 & 5'-AGGGATGTTTAATACCACTAC-3' \\
TADC1 & 5'-TTGATTGGAGACTTGACC-3' \\
UK-Seq-1 & 5'-CCCAACCAAGAAGTATGCTGA-3' \\
UK-Seq-2 & 5'-TCACAGGGAAGGAGACAATGGCA-3' \\
UK-Seq-3 & 5'-ATGAAATCATGTGTTGTTGTGGCA-3' \\
\hline
\end{tabular}

to sample buffer with yeast cells, and the reaction mixtures were incubated at $37^{\circ} \mathrm{C}$ for $15 \mathrm{~min}$. For freeze-thaw treatment, the yeast cells resuspended in sample buffer were frozen at $-80^{\circ} \mathrm{C}$ for 5 min and then thawed at room temperature for $5 \mathrm{~min}$. For amplification from glycerol stocks, $1 \mu \mathrm{L}$ of yeast stocks kept in $-80^{\circ} \mathrm{C}$ freezers was directly mixed with $5 \mu \mathrm{L}$ of sample buffer. All the samples with the above treatments were incubated at $95^{\circ} \mathrm{C}$ for $3 \mathrm{~min}$ and then cooled down to $4^{\circ} \mathrm{C}$. Five micro-

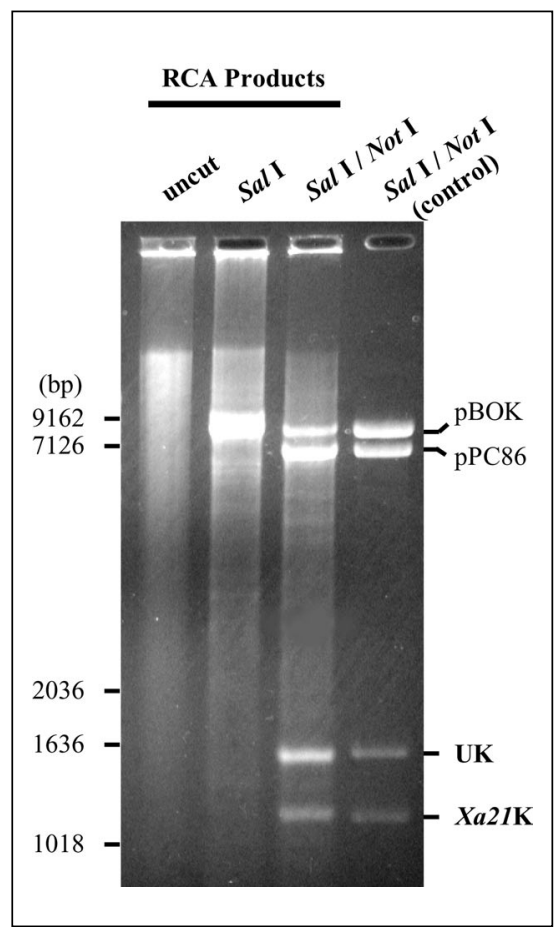

Figure 1. Diagnostic restriction digest and agarose gel electrophoresis of plasmids $\mathrm{PBOK}$ XA21K and pPC86-UK amplified from yeast cells by rolling circle amplification (RCA). Single yeast colonies were picked and amplified in $10-\mu \mathrm{L}$ volumes; $1 \mu \mathrm{L}$ of the amplified DNA was digested with the indicated enzymes. Restriction digestion of the pBOK-XA21K and pPC86-UK plasmids purified from Escherichia coli is also shown as control. liters of reaction buffer and $0.2 \mu \mathrm{L}$ of the $\$ 29$ DNA polymerase (Amersham Biosciences) were added to the denatured cell suspensions. The mixtures were incubated at $30^{\circ} \mathrm{C}$ for $6-18 \mathrm{~h}$. Restriction digestion of the RCA products was performed in $15-\mu \mathrm{L}$ volumes. One microliter of amplified DNA was incubated with restriction enzymes in the appropriate buffer at $37^{\circ} \mathrm{C}$ for $4 \mathrm{~h}$.

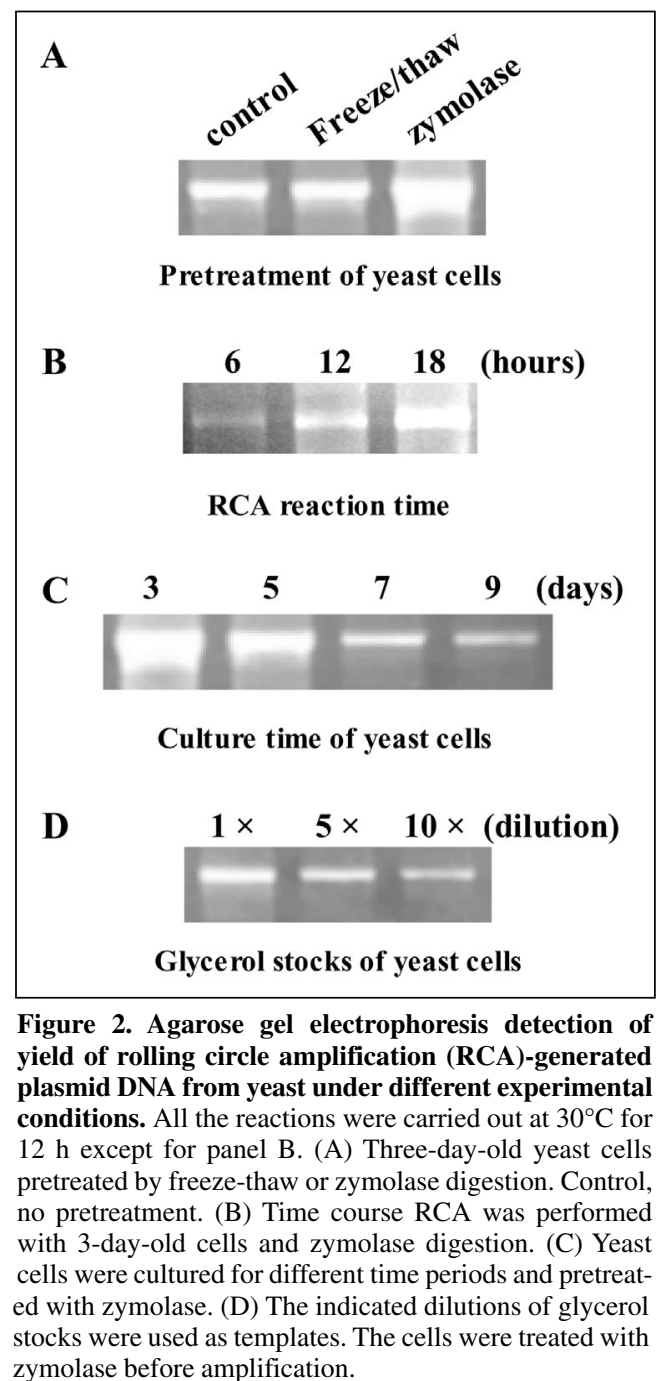

\section{RESULTS AND DISCUSSION}

We chose a yeast strain carrying both pBOK-XA21K and pPC86-UK plasmids to perform RCA. Three-dayold single colonies were picked directly from medium plates. After a rapid DNA isolation procedure (described below) and RCA for $12 \mathrm{~h}$ at $30^{\circ} \mathrm{C}, 1-3$ $\mu \mathrm{g}$ of DNA in a broad size range were amplified (Figure 1).

To confirm that the amplified products contained the template DNA, restriction digestion with the single-cut enzyme SalI was performed. A major band of $9 \mathrm{~kb}$ was obtained (Figure 1). Double-digestion with SalI and NotI created four bands that were identical in size to the products of $\mathrm{pBOK}-\mathrm{XA} 21 \mathrm{~K}$ and pPC86-UK cleaved by the same enzymes. These results indicated that the amplified DNA was concatemers of pBOK-XA21K and pPC86-UK. In addition to these two plasmids, which comprised the majority of amplified products, some background, presumably amplified from yeast chromosomal DNA, was also observed.

The template preparation and RCA reactions were optimized using yeast cells containing a single plasmid (pBOK-XA21K). Freeze-thaw treatments have been used to lyse yeast cells in $\beta$-galactosidase filter assays (7). However, these treatments did not result in an increase in the amount of amplified products (Figure 2A). In contrast, incubation of cells with zymolase, an enzyme that hydrolyzes glucose polymers of yeast cell walls at $\beta$ 1,3-glucan linkages, significantly increased the yields of RCA products. Following zymolase treatment, amounts of amplified DNA increased with RCA reaction time (Figure 2B). Six hours after RCA reaction, the amplified products can be detected by gel electrophoresis.

Amplification efficiency using yeast cells cultured for different time periods and cells from glycerol stocks was tested. Younger cells yielded better templates for RCA. A linear decrease in the amount of amplified prod- 
ucts was observed with an increase in culture time during the 9-day period examined (Figure 2C). For example, the yield from 3-day-old yeast cells is 10 -fold higher than that amplified from 9-day-old cells. Three days of growth were optimum for RCA. In addition, the pBOK-XA21K DNA was also directly amplified from $1 \mu \mathrm{L}$ of yeast glycerol stocks, which would facilitate high-throughput RCA using frozen samples (Figure 2D).

RCA DNA amplified from the yeast cells containing pBOK-XA21K and pPC86-UK was sequenced using a Dye terminator DNA sequencing kit (Amersham Biosciences). Up to 600 bases of high quality sequence were typically obtained in a single run (data not shown), indicating that RCA-amplified plasmids were excellent templates for DNA sequencing. By using with primer SSO20 specifically recognizing pPC86-UK or primer GAL4-DB recognizing $\mathrm{pBOK}-\mathrm{XA} 21 \mathrm{~K}$, the insert of each of the two plasmids can be

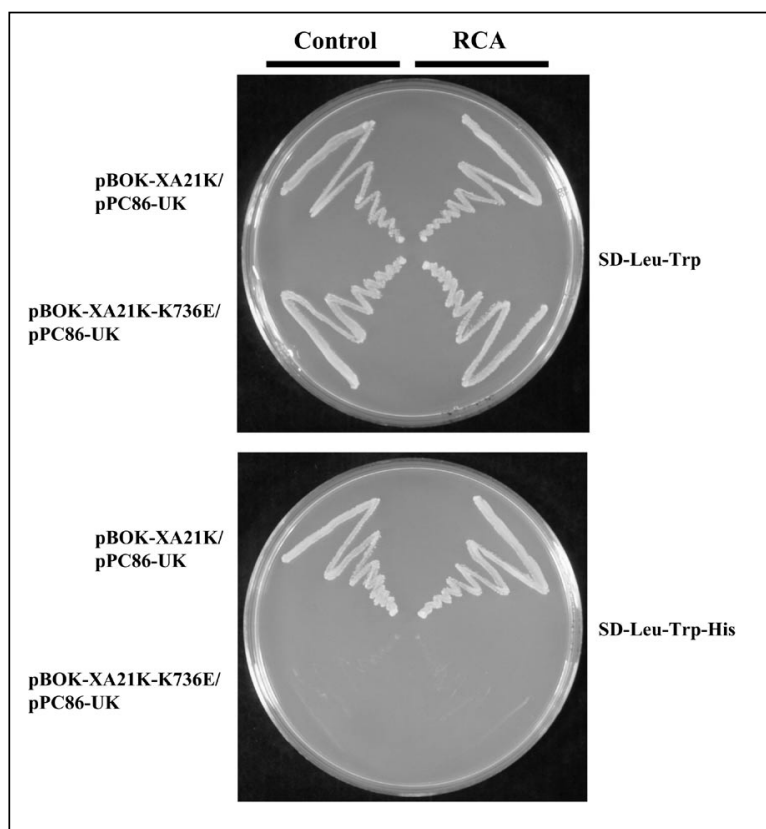

Figure 3. Yeast two-hybrid assays using rolling circle amplification (RCA)-amplified products. Yeast cells transformed with the indicated constructs were grown on the selective medium specified on the right. Colonies capable of growing on the SD medium without leucine and tryptophan show the presence of the transformed plasmids, whereas colonies growing on the SD medium lacking leucine, tryptophan, and histidine indicate activation of the reporter gene His 3 resulting from the interactions of the XA21K and UK fusion proteins in the yeast cells. Left side (control), the plasmid DNA used for transformation was isolated from Escherichia coli using conventional methods; right side (RCA), the DNA was amplified from the yeast cells carrying the indicated plasmids. selectively sequenced from the mixed RCA templates. These results make it possible to identify the interactors without physical separation from the bait constructs.

To estimate the error rate of RCA in our experiments, plasmid DNA isolated from 20 randomly $\mathrm{XA} 21 \mathrm{~K}$ and $\mathrm{pPC} 86-\mathrm{UK}$. The coding region of $X a 21 K(1042 \mathrm{bp})$ and $U K$ (1486 bp) was completely sequenced not shown). Only one mutation should not functional assays, because of transformants would likely be collectively used.

We have previously shown that XA21K interacts with UK in the yeast two-hybrid system, but not with an XA21K mutant, XA21K736E (L.-Y. Pi and W.-Y. Song, unpublished data). We then tested whether the RCA products can be used in the yeast two-hybrid assays. Two pairs of plasmids (pBOK-XA21K/pPC86-UK and pBOK-XA21K-K736E/pPC86$\mathrm{UK}$ ), prepared by RCA and by the conventional rescue methods, respectively, were transformed into yeast cells. By using standard procedures, an efficiency of $4.6 \times 10^{3}$ transformants/ $\mu \mathrm{g}$ DNA was achieved for the RCAamplified pPC86-UK. Typically, 0.5-1 $\mu \mathrm{g}$ of RCA products can produce sufficient amounts of transformants for subsequent analyses. Functional assays indicated that the RCA-amplified DNA functions similarly to its template in all the aspects tested, including $L E U 2$, TRP selections, and the XA21K-UKspecific interactions (HIS3 and LacZ) (Figure 3 and data not shown).

Yeast has very active homologous recombination systems (8). We reasoned that monomers of bait and prey could be created by homologous recombination from concatemeric RCA products. To confirm this hypothesis, we isolated plasmid DNA from 10 independent yeast colonies transformed with pPC86-UK using conventional methods, respectively. The rescued DNA was further propagated in Escherichia coli and then analyzed by gel electrophoresis. Figure 4 shows that the

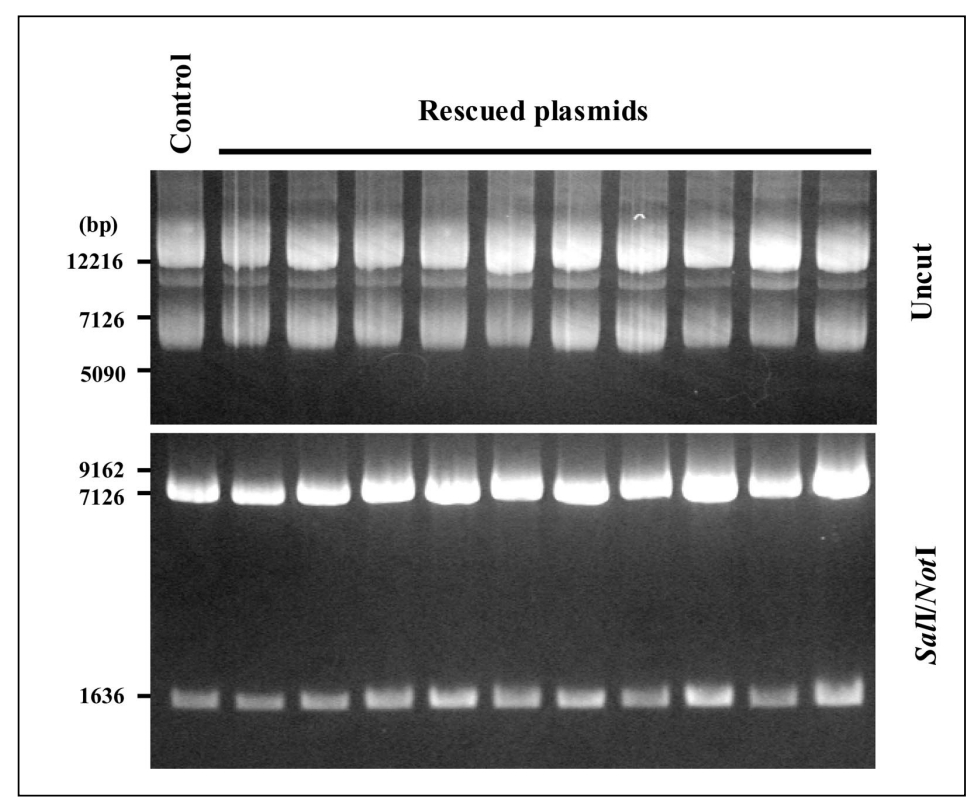

Figure 4. Agarose gel electrophoresis of the plasmids rescued from the yeast cells transformed with rolling circle amplification (RCA) products. Yeast cells were transformed with the RCA-amplified pPC86-UK DNA. Plasmids were recovered from 10 independent transformants, propagated in Escherichia coli, and analyzed by agarose gel electrophoresis. Both uncut (upper) and SalI-NotI digested (lower) samples are shown. Control, pPC86-UK. 
recovered plasmids are similar in size to the monomeric control construct. Restriction digestion confirmed that these plasmids contained the correct insert (Figure 4). Thus, yeast can generate monomeric circular plasmids from transformed RCA products.

The primary goal of the study was to develop a simplified procedure for conducting yeast two-hybrid analysis on a large scale. The RCA-based approach described in this study eliminates a number of laborious and time-consuming steps related to the propagation of plasmid DNA using E. coli as hosts in the conventional yeast two-hybrid procedures.

Compared with the PCR-mediated in vitro DNA amplification, the RCA system also has several advantages. It can efficiently amplify circular DNA ranging in size from small plasmids to bacterial artificial chromosomes with the size of several hundred thousand base pairs. The random hexamer primers are universal for the amplification of any plasmid, which eliminates the needs to synthesize gene-specific primers. Because RCA mainly utilizes original plasmids rather than newly synthesized DNA as a template for amplification, the error rate of amplification can be greatly reduced, and the contamination risk is minimal. More importantly, the capability of high-fidelity amplification of large pieces of DNA containing multiple genes enables the complex functional assays requiring a cooperative action of multigene products. These features make the RCA products amenable to yeast two-hybrid analysis, particularly on a large scale.

In general, the RCA-based approach to manipulating plasmid DNA in yeast is simple, reliable, and robust. It is based on standard laboratory equipment and can be easily carried out in a high-throughput manner. The approach was originally designed to simplify the recovery of plasmids from large-scale yeast two-hybrid projects. However, this RCA approach could be used in other yeast-based systems, such as yeast one-hybrid, reverse yeast two-hybrid, and yeast three-hybrid screenings, thereby facilitating the utility of yeast as a tool for molecular biology and genomics research.

\section{ACKNOWLEDGMENTS}

We thank Michael Fromm and Dean Gabriel for critical reading of the manuscript and invaluable comments on the work. This research was supported by the Florida Agricultural Experiment Station and grants from the National Science Foundation Plant Genome Research (NSF-PGR) and the U.S. Department of Agriculture (USDA) to W.-Y.S. This work was approved for publication as Journal Series No. R-09518.

\section{REFERENCES}

1.Brent, R. and R.L. Finley, Jr. 1997. Understanding gene and allele function with two-hybrid methods. Annu. Rev. Genet. 31: 663-704.

2.Fire, A. and S.-Q. Xu. 1995. Rolling replication of short DNA circles. Proc. Natl. Acad. Sci. USA 92:4641-4645.

3.Dean, F.B., J.R. Nelson, T.L. Giesler, and R.S. Lasken. 2001. Rapid amplification of plasmid and phage DNA using phi29 DNA polymerase and multiply-primed rolling circle amplification. Genome Res. 11:1095-1099.

4.Blanco, L., A. Bernad, J.M. Lazaro, G. Martin, C. Garmendia, and M. Salas. 1989. Highly efficient DNA synthesis by the phage phi29 DNA polymerase. Symmetrical mode of DNA replication. J. Biol. Chem. 264: 8935-8940.

5.Garmendia, C., A. Bernad, J.A. Esteban, L. Blanco, and M. Salas. 1992. The bacteriophage phi29 DNA polymerase, a proofreading enzyme. J. Biol. Chem. 267:2594-2599.

6.Esteban, J.A., M. Salas, and L. Blanco. 1993. Fidelity of phi29 DNA polymerase. Comparison between protein-primed initiation and DNA polymerization. J. Biol. Chem. 268:2719-2726.

7.Walhout, A.J. and M. Vidal. 2001. Highthroughput yeast two-hybrid assays for largescale protein interaction mapping. Methods 24:297-306

8.Szostak, J.W., T.L. Orr-Weaver, R.J. Rothstein, and F.W. Stahl. 1983. The doublestrand-break repair model for recombination. Cell 33:25-35.

Received 19 June 2003; accepted 16 July 2003.

Address correspondence to Wen-Yuan Song, Department of Plant Pathology, Plant Molecular and Cellular Biology Program, University of Florida, Gainesville, FL 32611,USA.e-mail:wsong@ifas.ufl.edu 